\title{
Editorial: Coastal Risk: Shores and Deltas in Peril
}

\author{
Clara Armaroli ${ }^{1 *}$, Derek W. T. Jackson ${ }^{2}$, Denise J. Reed ${ }^{3}$ and Christophe Viavattene ${ }^{4}$ \\ ${ }^{1}$ Department of Physics and Earth Sciences, University of Ferrara, Ferrara, Italy, ${ }^{2}$ School of Geography and Environmental \\ Sciences, Ulster University, Coleraine, United Kingdom, ${ }^{3}$ Pontchartrain Institute for Environmental Sciences, University of \\ New Orleans, New Orleans, LA, United States, ${ }^{4}$ Flood Hazard Research Center, Middlesex University of London, London, \\ United Kingdom
}

Keywords: coastal, erosion, flooding, storms, restoration, sea-level rise, morphodynamics, ecosystem services

Editorial on the Research Topic

\section{Coastal Risk: Shores and Deltas in Peril}

Coastal systems are the result of a natural equilibrium between hydrodynamic, atmospheric, and terrestrial parameters and sediment dynamics. In the Anthropocene, this equilibrium in many coastal regions can be altered by human activities. These activities may globally magnify the effects of extreme meteorological events and sea level rise and directly influence coastal processes down to a local scale within and between river catchments, the sea, and the coast. While most interventions, such as urban development, seawalls, and jetties are placed for specific human benefits, their indirect effects on coastal economies, societies and ecosystems can be significant.

This Research Topic brings together research from across the world to illustrate the dramatic and diverse nature of the peril that coasts and deltas face. Scientific understanding of the dynamics of these systems is essential to their current management and for the development of adaptation strategies to reduce future risk in the face of climate change.

The dynamics of beach environments and the role of storms is the focus of several papers. Guisado-Pintado and Jackson note that more frequent and more intense Atlantic storms over the last 40 years have heightened the potential risk to coastal environments, population, and infrastructure. They examined the effects of two storms on a beach system in NW Ireland and conclude that that storm energy is not always a direct indicator of coastal impact. Local forcing factors and antecedent beach conditions can be more important drivers of coastal response on sandy beaches. Storm waves from "nor'easters" which impact the New England (USA) coast during winter and early spring are a key influence on the Plum Island system studied by Hein et al. using beach surveys, sediment sampling, numerical modeling, and historical analysis. They found a migrating "hot spot" of erosion which presents challenges for local communities.

The interaction of local beach dynamics and sediment supply with larger scale, longer time scale changes imposed by migrating Amazon mudbanks is the focus of two papers. Jolivet et al. found that multi-decadal beach mobility reflects influences on the wave regime from alongshoremigrating banks (strong wave dissipation and limited beach mobility) and inter-bank areas (limited wave dissipation and larger beach mobility). Periods of erosion from these multi-scale interactions impact local communities by reducing beach space available for recreation and turtle-nesting. The beach has also been influenced by commercial sand mining (Anthony et al.), exacerbating the effect of natural sand "sinks" on downdrift areas. The importance of these beaches for turtle nesting is noted in both papers, highlighting important ecosystem effects from human impacts.

Armaroli C, Jackson DWT, Reed DJ and Viavattene C (2019) Editorial:

Coastal Risk: Shores and Deltas in

Peril. Front. Earth Sci. 7:323.

doi: 10.3389/feart.2019.00323 
Pressures of human activity extend into estuaries where the interaction of riverine and tidal forcing on sediment dynamics governs the ability of wetlands to keep pace with sea-level rise. In the Sundarbans (Bangladesh), extensive mangroves forests are fed by a complex system of tidal channels that distribute river sediments. Hale et al. found that while increased sea levels, and associated increased in flooding frequency within the mangroves, could bring more opportunity for sediment deposition, sediment supply to the system is threatened by a proposed upstream project that could decrease the sediment loads of the Ganges and Brahmaputra Rivers by as much as 75 and 25\%, respectively. Maintaining systems like the Sundarbans is important for a storm protection role, and the same issue drives restoration of estuarine wetlands elsewhere. In NW Europe, many tidal wetlands have been drained for agricultural use, often leading to land subsidence. Efforts are underway to re-introduce the tide in some "set-back" schemes to regain ecosystems and provide storm buffers to coastal protection works. Brunetta et al. examined the morphological evolution and sediment distribution on one such scheme in SW Netherlands, projecting a 8-10 years period for marsh vegetation to establish and perhaps 50 years before a mature marsh forms.

Such time scales for restoration make it important to retain coastal features that provide vital services, such as storm protection for coastal communities. Coral reefs are a good example. Shope and Storlazzi used numerical modeling to show how the morphology of atolls influences sediment transport. With SLR, all shorelines exhibited an intensification of preexisting erosion/accretion patterns. Atoll islands most susceptible to shoreline instability with SLR are narrow, located on small atolls, with narrow and deep reef flats. Loss of reefs can have important implications, especially in tourism areas subject to coastal storms. The Mesoamerican Reef provides risk reduction benefits in Quintana Roo for human infrastructure. The annual risk reduction benefits are estimated at 4,600 people, USD 42 million damage prevention for buildings, and 20.8 million USD for hotel infrastructure (Reguero et al.). Such valuation reiterates the need to protect and maintain natural infrastructure.

The interaction between people and the coast is multifaceted and understanding dynamic feedbacks is important at a number of scales. Such complexity, however, is challenging to simply summarize so that it can be used at times of need. Response to oil spills is one example where, under emergency situations, knowledge of coastal dynamics is needed to guide clean up activities. The Adriatic Sea is a highly trafficked area for oil tankers and mixed sand and gravel beaches need to be understood to inform response planning. Grottoli and Ciavola noted that the dynamics of storm berms on these beaches lead to the potential for rapid oil burial, and discuss how such information could be better incorporated into oil spill response planning. Geomorphic complexity is further complicated by social and economic activities. Armaroli et al. use hazard modeling combined with an impact assessment model to quantify direct and indirect impacts of storm events on two villages in northern Italy. By considering the effects on people, property, and businesses the combined modeling approach was able to identify which village was subject to greater overall risk, enabling improved planning of coastal defense measures.

Several of these papers illustrate the current focus on the potential impact of coastal hazards on property and infrastructure. Cooper and Jackson argue that this has distorted approaches to assessing and managing physical coastal systems. They propose a five-category simple assessment of "coastal health" that could be used to assess the potential for returning to a naturally functioning physical coastal system.

Whether the realization that coasts and deltas are in peril is primarily driven by risk to human activities likely varies from system to system. The value of natural coastal systems is clear, however, and collectively these papers illustrate the process complexity and interacting scales of morphodynamics, across a variety of coastal settings, that must be considered for that "peril" to be managed and, if possible, mitigated.

\section{AUTHOR CONTRIBUTIONS}

DR wrote the main text with editing and additional contributions from DJ, CA, and CV.

Conflict of Interest: The authors declare that the research was conducted in the absence of any commercial or financial relationships that could be construed as a potential conflict of interest.

Copyright (C) 2019 Armaroli, Jackson, Reed and Viavattene. This is an open-access article distributed under the terms of the Creative Commons Attribution License (CC $B Y)$. The use, distribution or reproduction in other forums is permitted, provided the original author(s) and the copyright owner(s) are credited and that the original publication in this journal is cited, in accordance with accepted academic practice. No use, distribution or reproduction is permitted which does not comply with these terms. 